\title{
Now we are five
}

\section{Nature Physics is five years old. So we're celebrating, with a bumper issue and an online special.}

Doesn't time fly? It hardly seems possible, but it is now five years since the launch of Nature Physics.

2005 seemed a particularly appropriate year for our launch - it was the international 'year of physics', marking the centenary of the publication of Einstein's seminal papers on Brownian motion, the photoelectric effect and special relativity. The year was packed with events, broadly cultural as well as specifically scientific, that celebrated Einstein's legacy in particular and physics in general.

On 1 October 2005, Nature Physics joined the party. Our first issue established the mix of research, reviews and comment that has characterized the journal ever since. Five years on, we've been taking a look back over the papers we've published. It's fascinating to see how certain fields have developed in that time, and even that some currently 'hot' topics didn't exist five years ago. This is the inspiration for our anniversary 'focus' which you can see online at www.nature. com/nphys/focus/5th-anniversary/ - a compilation of the material we've published on the hottest of these topics over the past five years.

The first of our chosen categories is 'exotic matter', in which graphene is undoubtedly a star player. In 2004, just before the launch of Nature Physics, Andrei Geim and colleagues hit on a straightforward way to make graphene, launching a burgeoning field of research. As well as its potential device applications, this remarkable two-dimensional material has thrown open all sorts of explorations of pure-physics phenomena, such as the Klein paradox and an unconventional quantum Hall effect. A new curiosity, the topological insulator, is now also making its mark, and is featured alongside other exotica from our pages, such as magnetic monopoles, supersolids and Wigner crystals.

'Cold' is also hot - our second category is dedicated to the study of atoms and molecules cooled to temperatures close to absolute zero. Interesting quantum effects appear in ultracold matter, such as the trimer states predicted in the 1970s by Vitaly Efimov that have now been seen in ultracold gaseous alkali atoms. Indeed, ultracold matter has become a useful tool in the exploration of few-body physics, and indeed many-body physics.

Since 2005, we've also been following the development of ideas in pursuit of a mechanism for high-temperature superconductivity: although guided by ever-improving data, there is still no clear consensus. But a new category of superconductor, layered iron-based materials, has burst onto the scene who would have expected a magnetic element such as iron to figure in a hightemperature superconductor? It's certainly a development that has enlivened the debate on this topic.
So there's no definite answer yet on high-temperature superconductivity, and neither is there a clear winner in the race to build the first large-scale quantum computer. But, as quantum effects are harnessed for the storage, processing and transmission of information, a wealth of experimental and theoretical advances in the field are in turn providing a fuller foundational understanding of quantum mechanics itself. All things quantum make up the fourth category of our anniversary focus.

Finally, we've highlighted some favourite items from the rest of our content - we've restricted ourselves to only a small selection, but it ranges from space to spintronics, the Big Bang to biophysics. That last has certainly been a growing topic over the past five years, as the relevance of physics in understanding and modelling biological processes becomes ever clearer. This month, we're publishing a Nature Physics 'insight' on physics and the cell - turn to page 723 for our collection of Review and Commentary articles exploring viruses, tumours, protein folding and neural dynamics.

The editorial team is proud to have reached the milestone of this fifth anniversary. Thanks is due to all of our authors and reviewers, whose ongoing and much appreciated - efforts have made the journal what it is. But is it what you want? We are always happy to receive your feedback, let us know what you think.

And here's to the next five years!

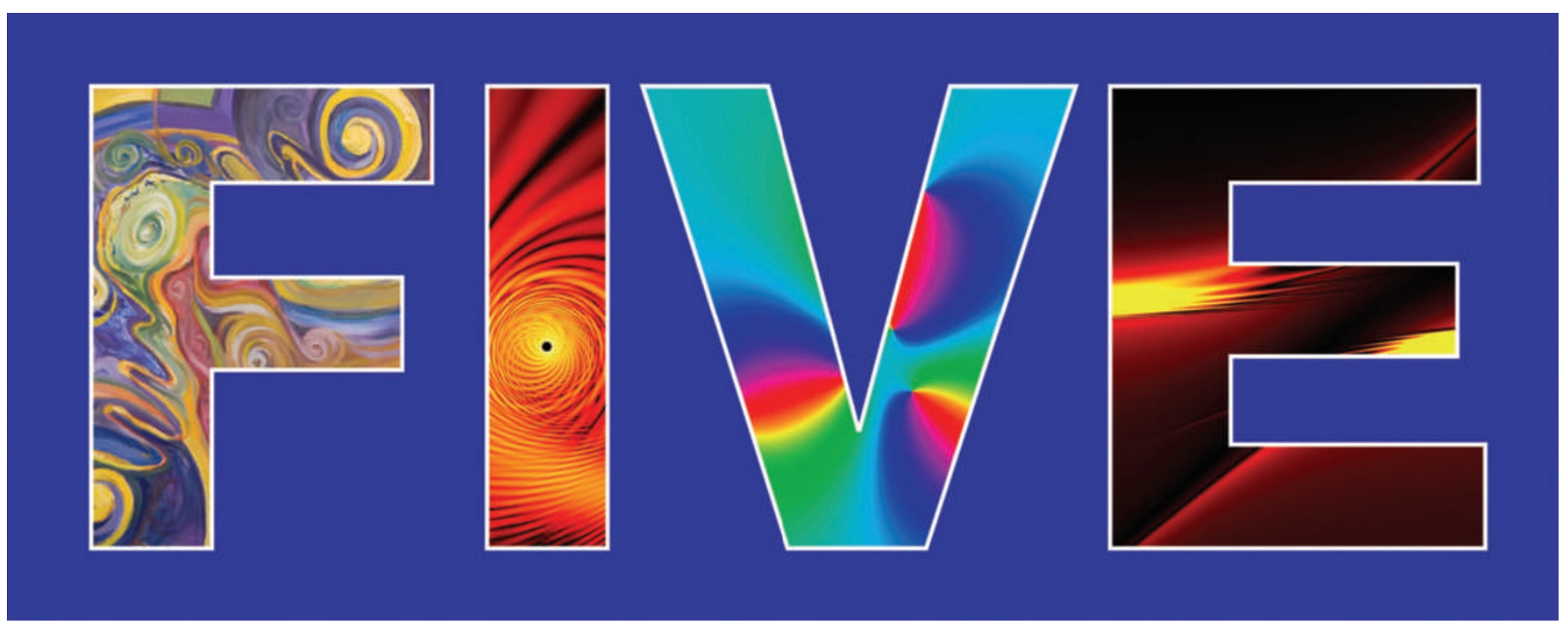

